Revue de recherche interdisciplinaire sur le genre et la sexualité

35 | 2018

Striges en tous genres

\title{
Etudier l'Etat au prisme du genre : réflexions éthiques et méthodologiques transatlantiques
}

Sabrina Calandrón, Laura Odasso et Maïté Maskens

\section{OpenEdition}

Journals

Édition électronique

URL : https://journals.openedition.org/sextant/378

DOI : $10.4000 /$ sextant.378

ISSN : 2795-8736

Éditeur

Éditions de l'Université de Bruxelles

\section{Édition imprimée}

Date de publication : 1 décembre 2018

Pagination : $37-54$

ISBN : 978-2-8004-1636-6

ISSN : $1370-267 X$

Référence électronique

Sabrina Calandrón, Laura Odasso et Maïté Maskens, « Etudier l'Etat au prisme du genre : réflexions éthiques et méthodologiques transatlantiques », Sextant [En ligne], 35 | 2018, mis en ligne le 01 novembre 2021, consulté le 08 décembre 2021. URL : http://journals.openedition.org/sextant/378 DOI : https://doi.org/10.4000/sextant.378

\section{(c) (i) (2)(2)}

La revue Sextant est mise à disposition selon les termes de la Licence Creative Commons Attribution Pas d'Utilisation Commerciale - Partage dans les Mêmes Conditions 4.0 International. 


\title{
Etudier l'Etat au prisme du genre : réflexions éthiques et méthodologiques transatlantiques
}

\author{
Sabrina CALANDRón, Laura OdAsso et Maïté MASKEnS
}

\section{Introduction}

Nous nous proposons ici de réfléchir aux pratiques et à la production de normes qui régissent les « rencontres étatiques ». Cette expression renvoie aux situations qui confrontent les citoyens ${ }^{1}$ nationaux et les migrants aux agents de l'Etat. Lors des procédures bureaucratiques, administratives ou judiciaires, l'Etat est loin de constituer une entité abstraite. Il est incarné par des acteurs institutionnels qui le représentent et qui, par leur travail, transmettent, produisent et, parfois, réinventent ou redéfinissent les lois et les normes ${ }^{2}$. Dans cette perspective, c'est le visage humain de l'Etat préoccupé par le contrôle des frontières nationales ${ }^{3}$ et ses implications genrées que nous aurons à cœur d'explorer grâce à la mise en perspective réflexive de trois terrains ethnographiques.

Les réflexions méthodologiques et théoriques livrées dans ces pages sont le fruit d'une collaboration scientifique entre l'Argentine et la Belgique autour du projet intitulé

${ }^{1}$ Dans le texte, les termes « citoyen » et « étranger » seront utilisés au masculin pour faciliter la lecture, toutefois ils sont à entendre dans la double acception féminine et masculine lorsqu'une acception féminine n'est pas spécifiée.

${ }^{2}$ Voir A. Buffat, M. Hill, P. Huppe (dir.), Understanding Street-Level Bureaucracy, Bristol, Policy Press, 2015 et M. LiPsky, Street-Level Bureaucracy : Dilemmas of the Individual in Public Services, New York, Russell Sage Foundation, 2010. Plus précisément sur l'immigration : A. SPIRE, Accueillir ou reconduire. Enquête sur les guichets de l'immigration, Paris, Raison d'Agir, 2008.

${ }^{3}$ Nous entendons ici à la fois les frontières extérieures (borders) et intérieures (boundaries) des sociétés étudiées. Voir D. FASSIN, "Introduction. Frontières extérieures, frontières intérieures ", in D. Fassin (dir.), Les nouvelles frontières de la société française, Paris, La Découverte, 2010, p. 5-24. 
State Encounters over the World : a Transatlantic Comparison of Professional Norms and Practices of the Polices and Bureaucratic Agencies related to Migration Policies et mené à bien par l'Universidad Nacional de Quilmes et de La Plata et l'Université libre de Bruxelles de 2015 à 2017. Loin de prétendre à une méthode comparative générale, au vu des différences historiques et sociales entre les deux pays ${ }^{4}$, nous plaidons pour une construction de l'objet par l'entremise de la comparaison ${ }^{5}$. La contextualisation et les descriptions particulières des sujets d'étude permettent d'orienter la comparaison et de mettre en lumière le dynamisme propre de phénomènes sociaux et culturels. Les auteures de cet article partagent un même intérêt pour l'étude des agents de l'Etat; elles participent ainsi à un mouvement de recherche centré sur les acteurs de pouvoir - dont la manière de concevoir le monde et de prendre des décisions en conséquence aura un impact sur la vie d'autres individus - à rebours de la tendance socio-anthropologique qui consiste à privilégier l'étude des acteurs dépourvus de pouvoir (les marginaux, les « primitifs », les victimes, les pauvres, etc. ${ }^{6}$. Sabrina Calandrón travaille sur les pratiques de contrôle et les valeurs professionnelles de la police en Argentine ${ }^{7}$. Laura Odasso examine l'Etat et ses politiques migratoires tels que les expérimentent les deux membres des couples binationaux hétérosexuels (homme et femme ; national et étranger) et les « intermédiaires du droit » qui les soutiennent dans leurs démarches administratives en France, en Belgique et en Italie ${ }^{8}$. Maïté Maskens suit le travail des

${ }^{4}$ Pour un approfondissement historique et social de l'histoire de la migration dans l'Etat argentin, voir O. OszLaK, La formación del Estado Argentino, Buenos Aires, Ariel, 2012 et M. BJERG, Historias de la inmigración en la Argentina, Buenos Aires, Edhasa, 2009. En ce qui concerne l'Etat belge, voir X. MABILLE, Nouvelle histoire politique de la Belgique, Bruxelles, CRISP, 2011 et pour les particularités de son histoire migratoire, voir M. MARTINIELLO, A. REA et F. Dassetto (dir.), Immigration et intégration en Belgique francophone. Etat des savoirs, Louvain-la-Neuve, Bruylant-Academia, 2007.

${ }^{5}$ A. Yengoyan, Modes of Comparison. Theory and Practice, Ann Arbor, The University of Michigan Press, 2006.

${ }^{6}$ L. NADER « Up the Anthropologist : Perspectives Gained from « studying up » », in D. Hyms (éd.), Reinventing Anthropology, New York, Random House, 1969, p. 284-311.

${ }^{7}$ Elle explore plus précisément les moralités qui guident le travail quotidien de la police et les enjeux de genre déployés entre collègues policiers, d'une part, et entre la police et la population sur laquelle elle agit, de l'autre. Elle se demande en particulier comment les femmes et des symboles féminins sont intégrés dans une profession historiquement occupée par des hommes. Voir S. Calandrón, Género y sexualidad en la policía bonaerense, San Martín, Unsam Edita, 2014.

${ }^{8}$ Elle cherche entre autres à appréhender les interactions entre couples et apparat étatique à la suite de la demande de mariage jusqu'à l'obtention d'un titre de séjour stable ou de la nationalité, et à décrypter les rôles joués dans ce contexte par les "intermédiaires du droit », notamment des acteurs associatifs et des experts juridiques, vis-à-vis des couples qu'ils soutiennent et vis-à-vis des politiques et des pratiques étatiques à l'œuvre. Elle a aussi interrogé des policiers et des fonctionnaires opérant les contrôles sur les unions (avant et après le mariage). Voir son texte dans le présent volume, « Le genre de l'engagement pour la défense du droit à la vie familiale en France »; L. ODAsso, «L'action des groupes militants en faveur des couples binationaux. Entre soutien humain, juridique et conscientisation », Recherches familiales, 14, 2017, p. 119-133 et ID. (dir.), Migration, amour et Etat : un ménage à trois, numéro spécial de la Revue de l'Institut de Sociologie, 2015/1-4 ; ID., " Views of Europe: 
agents de l'Etat chargés de la lutte contre les mariages de complaisance ${ }^{9}$ dans des communes de la ville de Bruxelles en Belgique pour comprendre les ressorts de leur décision ${ }^{10}$.

Nos recherches ont aussi en commun de se consacrer à l'analyse de l'Etat « depuis l'intérieur ${ }^{11}$, et ce, tant en termes méthodologiques, notamment qualitatifs, qu'en termes d'analyses. Dans nos travaux et à la suite de penseurs de l'Etat comme Didier Fassin et d'autres, nous ne comprenons pas l'Etat comme une entité homogène et abstraite, mais bien comme un objet multiforme et changeant qui s'incarne dans le travail de ses agents. En effet, les asymétries de pouvoirs et les formes de complicité entre les représentants de l'Etat et les populations à qui ils s'adressent suggèrent ce polymorphisme de l'Etat. Les actions étatiques prennent du sens au regard du déploiement de politiques particulières, de savoirs experts, de trajectoires éducatives, de techniques et de sujets qui les amorcent. Ces actions s'expliquent aussi en partie au travers des tensions inhérentes aux interactions entre usagers et agents ainsi qu'à la lumière de logiques collectives particulières. En adoptant cette approche, nous désirons rendre compte dans ce qui suit des dynamiques et des formes d'organisations internes à l'Etat au-delà de la réduction conceptuelle qu'imposerait une approche mécanique, homogène ou identique de reproduction dans le développement de ses activités quotidiennes. L'appareil étatique développe des conceptions et des représentations variées, parfois stigmatisantes, de la circulation des personnes et des biens sur la base desquelles il fonde les critères permettant de positionner la frontière entre ce qui est légitime, conforme ou légal, et ce qui ne l'est pas. En outre, sur la base de la légitimité de son propre pouvoir, l'Etat définit des catégories lui permettant de déployer son contrôle et sa protection. Des logiques parfois contradictoires, et souvent discriminantes, peuvent coexister au sein de l'Etat ${ }^{12}$. Nous étudierons leurs effets en focalisant notre attention sur les pratiques du genre.

Dans la première partie de cet article, nous explorerons le genre et ses liens avec le concept de « famille » dans les pratiques étatiques. Dans la seconde partie, nous aborderons le genre de manière transversale à partir de nos trois terrains ethnographiques en nous arrêtant sur les implications méthodologiques et éthiques d'une telle approche. L'apport innovant de ce travail réside dans la mise en lumière

National Civil Society Organisations for Binational Family Rights on the Road to Brussels ", Journal of Contemporary European Research, 14/2, 2018.

${ }^{9}$ Que celui-ci implique les deux personnes qui échangent un avantage de séjour contre une rémunération - il s'agit alors d'un mariage blanc -, ou qu'il concerne seulement la personne étrangère du couple qui dupe l'autre - on parle alors de mariage gris. Voir M. SALCEDO RoBLEDo, « Bleu, blanc, gris... la couleur des mariages », L'Espace politique, 13/1, 2011 (https://espacepolitique.revues.org/1869).

${ }^{10}$ Voir M. MAsKens, « Bordering Intimacy : The Fight against Marriages of Convenience in Brussels », Cambridge Journal of Anthropology, 33/2, 2015, p. $42-58$ et ID. (dir.), Migrations Société, dossier spécial : Mariages et migrations : l'amour et ses frontières, 150/25, 2013.

${ }^{11}$ E. BohosLavsky et G. Soprano, Un Estado con rostro humano, Buenos Aires, Prometeo y Universidad Nacional de General Sarmiento, 2010 ; D. Fassin (dir.), Juger, réprimer, accompagner. Essai sur la morale de l'Etat, Paris, Seuil, 2013.

${ }^{12}$ A. SAYAD, "Immigration et " pensée d'Etat » ", Actes de la recherche en sciences sociales, numéro thématique : Délit d'immigration, 129, 1999, p. 5-14. 
d'implications éthiques similaires qui émergent de l'observation d'espaces politiques et administratifs différents - et rarement comparés. En effet, l'enquête ethnographique montre le double lien entre le genre du chercheur et le genre de l'Etat, entre investigation et réflexivité socio-anthropologique. Le défi est donc de donner à voir le pouvoir de l'Etat en ne se focalisant pas seulement sur les mécanismes de l'exclusion, mais en donnant également à penser les mécanismes inclusifs et propositionnels du pouvoir ${ }^{13}$. Nous nous appuierons sur des extraits de notes de terrain et des extraits d'entretiens pour donner corps à nos propos.

\section{Observer les notions de genre et de famille au sein de pratiques étatiques}

Les relations de genre participent à la mise en œuvre quotidienne des politiques publiques et une certaine idéologie genrée produit des « cadres moraux » ${ }^{14}$ qui guident les logiques et les interventions des agents étatiques et qui, par ricochet, affectent les attitudes, les émotions et les comportements des individus concernés et leurs familles. La famille et son contrôle sont au cœur de la gestion de l'appartenance à la communauté ou, plus récemment, à la nation ${ }^{15}$. En effet, la définition des modèles et des rôles familiaux qui découle des textes législatifs et des circulaires administratives a une incidence déterminante sur la conception pratique et quotidienne genrée des politiques publiques et sur la formation professionnelle des agents étatiques - hommes et femmes - chargés de les mettre en œuvre. Ce que « l'Etat fait à la famille » ${ }^{16}$ est donc un sujet crucial lorsqu'on vise à appréhender la relation entre genre et Etat. Les modèles familiaux et les rôles genrés proposés par l'Etat, à travers son droit et ses politiques publiques, ont des conséquences contraignantes sur les vécus des individus et sur leur capacité d'agir qui nécessitent une observation attentive.

En Belgique, cela se traduit par des conceptions normatives de la conjugalité et des relations affectives qui constituent les critères implicites qui façonnent la mise en œuvre des politiques migratoires. Ces dernières circonscrivent ce qu'est une " bonne famille » dans le champ de la migration, sans considérer les changements dans les dynamiques conjugales et affectives existantes. Ainsi, il est demandé aux couples binationaux et/ou formés par des migrants de remplir des conditions qui ne sont pas requises pour les autres couples ${ }^{17}$. Ces couples binationaux doivent bien

13 M. AbÉLÈs et M. BADARó, Los encantos del poder. Desafíos de la antropología política, Buenos Aires, Siglo XXI, 2015.

14 D. Fassin (dir.), Juger, réprimer, accompagner, op. cit. et D. FASsin, J. S. Eideliman (dir.), Economies morales contemporaines, Paris, La Découverte, 2012.

15 N. Yuval-Davis, Gender \& Nation, Londres, Sage, 1997.

16 P. Bourdieu, «A propos de la famille comme catégorie réalisée ? », Actes de la Recherche en Sciences sociales, 100, 1993, p. 32-36.

17 S. Sarolea et L. Merla, « Migrantes ou sédentaires : des familles ontologiquement différentes ? », communication au colloque Migrations familiales et usages du droit, Marseille, 22-23 février 2018. Audio en ligne : migrationsfamiliales.wixsite.com/colloque (publication à paraitre ; M. MASKENS, "Secular selves and bodies : the case of state agents in charge of implementing the fight against marriages of convenience in Brussels », in J. MAPRIL, E. Giumbelli et R. Blanes (éd.), Secularism in a Postsecular Age? Religiosities and Subjectivities in Comparative Perspective and Subjectivities, Palgrave Macmillan, 2017, p. 17-38 ; ID., « Bordering Intimacy », op. cit. et ID. (dir.), Migrations Société, op. cit. 
souvent présenter un projet conjugal et familial sur la durée qui correspond à des formes conjugales repérables sur le territoire belge. En outre, le citoyen belge qui désire se marier ou conclure une cohabitation légale avec un ressortissant d'un pays tiers extra-européen doit désormais remplir les mêmes conditions qu'un ressortissant étranger qui souhaite entamer la même démarche. Ainsi, en raison du choix de son conjoint, le citoyen est " mis sur le même pied d'inégalité » qu'un étranger par les lois de l'Etat belge ${ }^{18}$. Sans compter qu'au-delà de ce que dit la loi, ces couples questionnés et suspectés sont condamnés, parfois pour des années, à la précarité légale et administrative ${ }^{19}$. Les pratiques administratives qui leur sont imposées se caractérisent par des déséquilibres genrés. Les femmes belges qui choisissent de se marier avec un étranger sont soumises à des rencontres étatiques visant à les mettre en garde contre les « risques » d'une mise en couple avec « l'autre » étranger, voire à les faire changer d'avis. Il est souvent rappelé à ces femmes le devoir de reproduire un certain type de nation ${ }^{20}$. En revanche, les hommes nationaux qui souhaitent se mettre en couple avec une ressortissante d'un pays tiers ne sont pas découragés comme leurs homologues féminins. Les femmes migrantes seront rarement considérées comme des sujets dangereux à introduire dans le tissu national, alors que les hommes étrangers sont plus souvent présentés comme constituant une menace (en fonction surtout de leur nationalité et de leur trajectoire migratoire). Le genre se décline de manière variable selon l'origine : l'union d'un Belge d'origine étrangère ou ayant des parents migrants sera considérée comme le signe d'un repli identitaire qui, au-delà du communautarisme, conduirait à des formes familiales plutôt homogames et inadaptées à la société belge. Ces stéréotypes, parfois contradictoires, se reproduisent à la fois dans les narrations des usagers et dans celles des agents. Dans leur travail quotidien, ces derniers opèrent sur la base de représentations de la famille et de rôles des hommes et des femmes qui façonnent la transmission des pratiques de contrôles dans les communes et dans les cellules de police. Les bureaux de l'administration sont eux aussi imprégnés des relations genrées qui modèlent les pratiques de contrôle. Ce n'est pas un hasard si la plus récente des cellules de police dite « mariage blanc »- qui contrôle la véracité des unions binationales lorsque les communes de la zone ne sont pas satisfaites des résultats de leurs enquêtes internes et ont saisi le procureur du Roi - n'est constituée que par des femmes. Ces policières ont été choisies en raison de leur parcours professionnel, mais aussi de leur sensibilité et de leur attitude plus empathique visà-vis des membres des couples. Ces caractéristiques semblent utiles pour décrypter les relations conjugales binationales à fin d'établissement sur le territoire, voire pour protéger ou mettre en garde des individus perçus comme vulnérables pris dans des relations binationales dont la police pense qu'ils ne saisissent pas les risques. On voit se dessiner ici les contours d'un Etat, proche d'une figure paternelle, qui a à cœur de protéger les femmes, perçues d'emblée comme vulnérables ou naïves.

${ }^{18}$ S. SAROLÉA, «Le regroupement familial suite à la réforme de 2011 », in S. BodART, Droit des étrangers, Bruxelles, Bruylant, 2012, p. 111-142.

${ }^{19}$ C. MAsCia et L. OdAsso, « Le contrôle du mariage binational en Belgique. Les règles du jeu », Revue de l'Institut de Sociologie, numéro spécial Migration, amour et Etat ; un ménage à trois, 2015/1-4, p. 47-68.

${ }^{20}$ N. Yuval-Davis, Gender \& Nation, op. cit. 
En Argentine, les études sur la police et les forces de sécurité permettent d'élargir la définition même de la famille ${ }^{21}$. Pour les agents de police de Buenos Aires, les conceptions de ce qu'est une "bonne famille " constituent un enjeu durant les interventions dans les secteurs socio-économiquement fragilisés de la population. Cela apparaît notamment lorsque des femmes sollicitent l'aide de la police parce qu'elles sont victimes de violences conjugales et qu'elles finissent par devenir elles-mêmes l'objet d'une évaluation, dans leur rôle de mère, par les policiers. Cette dynamique de contrôle qui se retourne contre les sujets demandeurs d'une présence de l'Etat, au nom de la « défense de la famille », est une constante que l'on retrouve dans le traitement des populations précarisées. Les critères d'évaluation morale qui pèsent sur les personnes défavorisées sont fortement liés au genre puisque c'est la maternité qui est en jeu, entendue sous sa forme la plus traditionnelle. Ce retournement n'apparaît pas lorsque les demandes d'intervention émanent d'individus appartenant à des secteurs plus prospères de la société. Ainsi, le terrain avec les forces de police argentines montre que les policiers ont une perspective particulière visant à appréhender les comportements des femmes et des hommes de manière différenciée pour évaluer la population et agir sur elle. Nous allons évoquer ici un épisode, qui relève à la fois du genre et de la famille, ayant eu lieu dans un commissariat qui traite prioritairement des cas de femmes et de familles.

Une femme de 28 ans, accompagnée par sa petite fille de 3 ans, s'approche pour dénoncer des maltraitances exercées par son compagnon. Dans les minutes d'attente préalables à son accueil, les agents ont noté que la femme ne s'occupait pas assez de son enfant qui grimpait sur les meubles, mettait les doigts dans les prises électriques et tentait de s'introduire dans des espaces étroits de la salle. En conséquence, les agents décideront qu'elle est une mauvaise mère et donc qu'elle ne mérite pas l'attention nécessaire. Du coup, elle a attendu plus que le temps prévu pour être reçue et, à ce moment-là, elle a été accusée verbalement de ne pas prêter l'attention requise à sa fille, ce qui rendait encore moins crédible sa déclaration par rapport à sa situation familiale (Carnet de terrain de S. CALENDRón, Buenos Aires, mai 2010).

Cette forme d'évaluation qui cherche à présenter les femmes comme des mères peu attentives se répétait tous les jours au fil du terrain de Sabrina Calendrón. Pour obtenir l'attention des agents, les femmes devaient démontrer être des mères qui consacrent tout leur temps à leurs enfants. Enfin, lorsque la chercheure leur demandait de préciser les crimes imputés aux femmes retenues dans le commissariat, les agents répondaient après avoir cherché dans leurs bases des données : infanticide (cela concerne à la fois les femmes qui ont tué leur enfant et celles qui ont avorté dans des situations et pour des motifs différents), homicide du mari, prostitution et aveu d'avoir couvert ou dissimulé des actes commis par leur mari - les plus «stupides ». Cette

${ }^{21}$ Voir L. CAimari, Mientras la ciudad duerme : pistoleros, policias y periodistas en Buenos Aires 1920-1945, Buenos Aires, Siglo XXI, 2012 ; M. SirimarCo, «A familia policial : Vinculações e implicações entre relato e instituição ", Mana, 19/3, 2013 (http://www.scielo.br/ scielo.php?script=sci_arttext\&pid=S0104-93132013000300006); S. CALANDRÓN, « La sagrada familia y el oficio policial : sentidos del parentesco en trayectorias y prácticas profesionales cotidianas ", in S. Frederic, J. Garriga Zucal, M. Galvani et B. Renoldi (éd.), De armas llevar, La Plata, Ediciones de Periodismo, 2013, p. 57-88. 
forme de catégorisation est liée en partie aux infractions du Code pénal et en partie aux codes moraux des agents de police. Les deux premières incriminations visent des femmes qui ont contrevenu au code de la maternité et de la famille, la troisième, celles qui ont violé le code de l'intimité, la quatrième, des femmes coupables de vol et d'escroquerie, mais qui, loin d'être vues comme actives, sont considérées par la police comme des personnes «stupides » ou « manipulables ». La passivité et la vulnérabilité des femmes vis-à-vis de l'escroquerie des hommes sont d'autres caractéristiques que les agents de police définissent et qui différencient la féminité de la masculinité dans la société civile.

De plus, avec le temps, en Argentine, la police et la gendarmerie se voient comme une famille au service d'une mission commune et d'une loyauté totale à l'institution policière. Ce qui conduit l'Etat à demander aux gendarmes de se sacrifier pour cette famille. Dans ces processus, les gendarmes finissent par consacrer moins de temps à leur propre famille, par exemple en raison de déplacements de longue distance. La nostalgie et le malaise que cela génère ne sont pas vraiment reconnus par la famille-gendarmerie qu'ils servent. De plus, et comme dans de nombreuses formes de reproduction familiales, ces institutions étatiques distribuent le pouvoir en fonction de deux critères : la préséance de l'âge (les pères exercent l'autorité sur leurs fils, les grands frères sur leurs petits frères, les aînés sur leurs cadets) et le genre (les hommes s'imposent aux femmes). La relation entre la famille et l'institution policière ne se limite pas à ce qu'on vient de citer. L'institution policière impose régulièrement un style de vie à ces agents : par exemple, les congés de maternité sont de trois mois, ceux de paternité, de deux jours ; tous les policiers, hommes et femmes, sont tenus d'informer leur supérieur lorsqu'ils se mettent en couple avec un autre policier et cette information est prise en compte pour la distribution territoriale du personnel (un couple ne devrait pas travailler dans le même édifice, mais pas non plus à plus de soixante kilomètres de distance). Pour les mariages entre agents de la même section, l'institution offre un cadeau de mariage et quinze jours de vacances pour le voyage de noces. Ainsi, de cette manière et parmi d'autres, l'Etat active des contrôles et des incitants vis-à-vis de la vie familiale des agents.

Dans le lieu de travail ou de formation, l'Etat active aussi des contrôles sur les relations entre hommes et femmes, et présuppose que les deux groupes d'individus nécessitent des services différents en raison des besoins qui seront naturalisés en raison du sexe. Par exemple, les écoles de formation des agents de la Gendarmería Nacional argentine ont des dortoirs séparés pour les hommes et les femmes. Les femmes dorment ensemble dans une grande chambre où les lits sont les uns à côté des autres. La structure est précaire et improvisée, mais deux éléments qui ont attiré notre attention différencient ces chambres de celles des hommes : dans les chambres des femmes, une sonnette allume une lumière rouge pour signaler la présence ou l'entrée d'un homme ; dans les sanitaires des femmes, un rideau de plastique sépare les douches. Ce que la sonnette et les rideaux démontrent, c'est la nécessité de préserver une certaine intimité physique des femmes par rapport aux hommes et entre elles, valeur qui ne semble pas importante pour les hommes. La virilité semble être reliée au fait de se montrer et de pouvoir être vu corporellement sans entrevaves. 
Et la féminité se réaffirme avec la pudeur qui préserve le corps des regards et de l'observation d'autres corps nus.

Les agents tant argentins que belges pensent les responsabilités propres des mères/ femmes/filles/épouses selon une terminologie différente de celles des hommes/pères/ maris/fils et cela influence leur travail et la manière dont ils traiteront leur public en fonction de leur sexe. Les conceptions liées au genre des usagers occupent donc une place centrale dans les pratiques étatiques, plus particulièrement lorsque les sujets de l'intervention de l'Etat présentent des situations familiales, conjugales ou amoureuses soumises à l'arbitrage des agents étatiques (bureaucrates et policiers). Ce sont eux qui évaluent la situation en se fondant sur des théories du genre implicites qu'ils activent dans des situations d'interaction étatiques que nous avons pu observer lors de nos recherches. Ces situations mettent en lumière les conceptions du genre en vigueur dans ces espaces de pouvoir qui sont structurées par 1) l'injonction à la maternité altruiste et responsable ; 2) la représentation d'une féminité vulnérable qui a besoin de la protection de l'Etat et d'une masculinité dangereuse ; 3) une féminité manipulable et facile à tromper.

A ces observations qui suggèrent la pertinence du genre dans l'objet d'étude en soi, c'est-à-dire au sein des rencontres étatiques que nous avons observées, s'ajoute le rôle imbriqué du genre dans la relation entre le chercheur et son objet d'étude. Ce deuxième aspect constitutif des investigations et des analyses génère des défis et des dilemmes moraux qui méritent d'être considérés dans le processus de recherche.

\section{Un parcours transatlantique réflexif : échanges méthodologiques et éthique du terrain}

Dans une perspective théorique visant à s'affranchir de l'unidirectionnalité dont souffre la communication entre des mondes académiques du Sud au Nord, un des objectifs du projet collaboratif d'échange belgo-argentin était de faire interagir des littératures latino-américaines et européennes. Si les chercheures argentines participantes au projet sont familiarisées avec une partie de la littérature européenne et anglo-américaine, les chercheures belges ont dû déplacer un regard souvent trop eurocentrique. Ces échanges théoriques ont été associés à des réflexions sur la posture de la chercheure, voire du « soi-chercheure » et du « soi-individu ». D'un point de vue méthodologique, sur le terrain, être une femme ou être un homme devient une entrée pertinente - parmi d'autres possibles (ethnicité, âge, classe...) - pour réfléchir à l'accès et à la production de données lors du travail de terrain concernant l'Etat ${ }^{22}$. Les relations genrées qui se développent dans l'interaction entre la chercheure et les hommes et les femmes observés et interrogés ouvrent ou ferment les accès à certains espaces de connaissances. C'est notamment la notion de perméabilité des interactions entre les individus étudiés et les chercheures qui a permis d'avancer dans la réflexion sur les nuances des résultats des recherches à propos des rencontres étatiques conduites en Argentine et en Belgique. Dans la mesure où l'ethnographie est un processus d'investigation basé sur la production d'un dialogue entre la perspective des

${ }^{22}$ M. Burawoy, « The Extended Case Method », Sociological Theory, 6/1, 1998, p. 4-33. 
ethnographiés et celle de l'ethnographe ${ }^{23}$, l'articulation entre ces deux perspectives est vitale pour produire de la connaissance. La perméabilité à laquelle on se réfère se concentre sur la rencontre entre ces perspectives divergentes et la possibilité d'incorporer les explications des ethnographiés dans nos analyses, emboîtant ainsi le pas à une co-construction du savoir socio-anthropologique.

\section{Etre une femme en terrain(s) étatique(s)}

L'Etat, tout comme les structures, les classes sociales ou le pouvoir en général, a d'abord été étudié par des hommes ou, pour le dire autrement, l'Etat fut abordé selon des perspectives androcentriques dont certaines furent réévaluées par la suite par des auteures féministes ${ }^{24}$. En effet, dès les années 1990, des chercheures ont souligné l'absence de prise en compte du genre dans les études sur les politiques sociales et la citoyenneté, attirant ainsi l'attention sur « le caractère heuristique d'une analyse genrée de l'Etat et de ses politiques ${ }^{25}$. En ce sens, d'un point de vue théorique, il n'est pas anodin de rappeler que les auteures et participantes au projet belgo-argentin étaient surtout des femmes ${ }^{26}$, contribuant ainsi à défaire cette division du travail intellectuel en sciences sociales. Chacune de nous pour son travail de terrain était plongée dans des lieux marqués par des formes particulières de pouvoir. Parfois même, comme dans le cas de Sabrina Calandrón, le terrain de recherche était un lieu de longue tradition masculine puisque les femmes n'intégrèrent le corps policier étudié par la chercheure argentine qu'à la fin du $\mathrm{Xx}^{\mathrm{e}}$ siècle. Le fait d'être une femme dans un milieu presque exclusivement masculin n'est pas sans conséquence sur la production de données ethnographiques. Afin de donner corps à ces affirmations, nous vous invitons à entrer dans les carnets de terrain de Sabrina Calandrón et à en lire deux extraits. Le premier a été réalisé dans un commissariat de la Police provinciale de Buenos Aires et le second se déroule dans un commissariat qui s'occupe des affaires familiales :

Je suis dans la cour intérieure du commissariat pendant qu'un des policiers, Alejandro (25 ans), peint la poutre d'une cellule. Sa collègue, Norma, une souslieutenante d'une cinquantaine d'années, lui tient compagnie de l'autre côté de la grille. Norma me fait participer à la conversation en me demandant si j'apprécie le sous-commissaire. Je suis surprise par sa question et je ne parviens pas à formuler une réponse claire à cette question : quelques mots incongrus sortent de ma bouche, car je ne veux pas manquer de politesse et je ne voudrais pas laisser penser que je suis attirée par le sous-commissaire. Norma continue : «Tu as un petit copain ? Ici on va

${ }^{23}$ M. Peirano, «A favor da etnografia », Série Antropologia, Brasilia, PPAS-UnB, 1992.

${ }^{24}$ N. Fraser, Fortunes of Feminism : From State-Managed Capitalism to Neoliberal Crisis, Londres, New York, Verso, 2013 ; N. Yuval-Davis, Gender \& Nation, op. cit. ; A. Phillips, Engendering democracy, Pennsylvania, Penn State University Press, 1991 ; C. Pateman, The Sexual Contract, Palo Alto, Stanford University Press, 1988 ; C. MacKinnon, Toward a Feminist Theory of the State, Massachusetts, Harvard University Press, 1989.

${ }^{25}$ B. Marques-Pereira et R. Pfefferkorn, «Introduction », Cahiers du genre, dossier spécial Genre, politiques sociales et citoyenneté : enjeux et recompositions, 3/2, 2011, p. 5-19 (p. 5).

${ }^{26}$ Outre les auteures de cet article, Barbara Truffin (juriste et anthropologue, Université libre de Bruxelles) et Sabina Frederic (anthropologue, Universidad Nacional de Quilmes) étaient parmi les principales participantes. 
t'en trouver un. Celui-là non [en pointant Alejandro du doigt] parce qu'il est vraiment idiot, mais ici, il y a beaucoup d'hommes, tu vas pouvoir choisir ! » (Carnet de terrain, Quilmes, mai 2010).

Dans ce fragment de travail de terrain, nous pouvons voir qu'en fonction du sexe de la chercheure et de l'attribution d'hétérosexualité qui est de mise, la femme policière l'inclut dans un type de relations pertinentes qui, selon sa perspective, prime dans ces espaces sociaux. Ainsi, Sabrina Calandrón accède à une idée en vigueur dans ces espaces particuliers : la sexualité est un bien apprécié dans les sphères policières et les femmes y occupent une position active. En même temps, cette situation expose le malaise, l'insécurité et l'expression de désir ou de non-désir dans le chef de la chercheure, car elle est une femme. Explorons maintenant un second extrait du carnet de terrain :

Au commissariat, une équipe interdisciplinaire s'occupe de réaliser des entretiens avec les personnes qui veulent recevoir des informations afin d'entamer une action en justice. Dans la majorité des cas, il s'agit de femmes qui viennent dénoncer leurs maris. L'agente féminine chargée considère que je peux être intégrée à cette équipe pour écouter le déroulement des entretiens : d'abord parce que je suis une femme et que l'équipe est constituée exclusivement de femmes (une psychologue, deux travailleuses sociales et une avocate) et puis aussi parce que je suis sociologue. Ce dernier point fait penser à l'agent que j'aurais un regard sensible sur ces réalités. C'est ainsi que je passerai trois heures à écouter les entretiens (Carnet de terrain, Quilmes, mai 2010).

Dans ce cas, « être une femme » permet à la chercheure d'entrer dans un lieu réservé aux pratiques d'Etat. Alors que dans le premier extrait, le genre de la chercheure renvoie à un intérêt pour la parade amoureuse, l'idéalisation du couple et de la sexualité, le second extrait montre que le fait que la chercheure soit une femme entraîne des associations avec une identité particulière qui relie les femmes à des valeurs spécifiques, par exemple l'écoute, la sensibilité et l'intégration à un groupe de femmes. Sans compter que toutes les femmes ne sont pas considérées de la même manière dans ces situations ; l'âge produit des « femmes différentes » comme le premier extrait le montre : Norma, une femme plus âgée qu'Alejandro et ayant dépassé l'état fertile, est libre de qualifier son collègue Alejandro d' ' idiot » sans provoquer de réaction de sa part.

Dans d'autres configurations étatiques, comme les Etats civils intégrés par Maïté Maskens, les employées étaient en large majorité des femmes. Cette dominance féminine influe à coup sûr sur l'« atmosphère » des bureaux, mais a aussi un impact sur les décisions prises par ces dernières. Le concept de « féminisme bureaucratique » développé dans ses travaux en est une illustration édifiante ${ }^{27}$. Dans ce contexte, le fait d'être une femme a permis un accès privilégié à la production de certains types de discours dans le chef de ses répondants masculins et féminins. Dans le cas des officiers masculins de l'état civil, ceux-ci inscrivaient leur mission de contrôleurs

${ }^{27}$ M. MASKENS, « Bordering Intimacy », op. cit. 
d'affects dans un « récit de sauvetage ${ }^{28}$ des femmes victimes de mariages gris, car dupées par des hommes migrants qui feignaient l'amour alors que leur seul objectif était d'obtenir un permis de séjour. C'est bien pour protéger ces femmes que l'appareil d'Etat aurait mis en place de nouveaux dispositifs de contrôle. Le cas d'hommes victimes de mariage gris n'a jamais été mentionné pour expliquer cette mobilisation politique. En ce qui concerne les agents féminins, la plupart du temps employées du bureau d'Etat civil, ce sont des idéaux d'égalité entre les sexes et de « liberté » dans l'architecture du choix du conjoint qui sont mis en avant pour expliquer leur décision d'accepter ou non les demandes de mariages. Les formes d'unions jugées comme non égalitaires ou machistes étaient refusées sur la base de leur incompatibilité avec les valeurs défendues dans ces espaces qui apparaissent alors comme des sites de frontières morales : le genre et la sexualité des migrants menaceraient l'espace européen aux mœurs libérées. Dans ce contexte, il n'est pas rare de voir un détournement de la législation sur les mariages de complaisance pour refuser des mariages arrangés, la forme retenue comme emblématique par les agents d'un mariage faisant fi de la liberté des individus et surtout de celle de la femme du couple. Pour donner à ces analyses une épaisseur empirique, plongeons-nous dans un « entretien mariage blanc » pour reprendre l'expression utilisée par les agents de l'Etat civil, entretien qui eut lieu au cours de l'année 2013 dans un bureau de l'Etat civil bruxellois entre deux agents de l'Etat, dont Martha, la chef de bureau et un couple belgo-albanais :

Occupée à retranscrire la réponse à la question précédente, Martha, agente de l'Etat en charge de la lutte contre les mariages de complaisance dans un bureau d'Etat civil bruxellois, demande à la femme albanaise de 32 ans assise en face d'elle, Alzana, quelles étaient ses attentes professionnelles en Belgique : « Et si votre mariage est accepté, vous comptez travailler? » Alzana répond que « oui », elle aimerait travailler comme aide-soignante, infirmière ou s'occuper des plus jeunes. Lorsque Martha posa la même question à son futur mari potentiel, Çelikan, un réfugié politique albanais et résident belge, celui-ci répondit, non sans fierté : «Non, ma femme ne travaillera pas comme ma mère, elle n'a jamais travaillé, elle s'est occupée de nous ». Lorsque le couple quitta les bureaux, les agents ont partagé leurs impressions sur ce qu'ils venaient d'entendre : la différence de réponse concernant le futur professionnel d'Alzana fut perçue comme la preuve d'une inégalité de genre dans le couple. La posture de l'homme " interdisant» à sa femme de travailler fut interprétée comme la marque d'une forme d'archaïsme patriarcale, le signe de la domination masculine. Martha clôturera le débat ainsi : "Que sera le futur de Bruxelles avec ce genre d'individus ? » et c'est sur la base de cet argument que le mariage fut refusé. Un peu plus tard, dans la même journée, un agent féminin vint me trouver pour partager avec moi le contenu d'un dossier qu'elle était en train d'examiner : " Regarde », me ditelle, « lorsqu'on a demandé à l'homme quelles étaient les qualités de sa future épouse, il nous a répondu « elle cuisine bien et elle passe l'aspirateur » et cela, vois-tu, ne nous paraît pas constituer les bases d'une vie commune alors on a refusé le mariage, mais le couple a fait appel et le tribunal leur a donné raison, car ils se connaissaient depuis longtemps » (Carnet de terrain, Bruxelles, 2013).

${ }^{28}$ L'expression « rescue narrative » est empruntée à S. BRACKE, « From « Saving Women » to "Saving Gays » : Rescue Narratives and their Dis/continuities », European Journal of Women's Studies, 19/2, 2012, p. 237-252. 
Le véritable enjeu dans cet extrait pour les agents n'est pas de contrôler la véracité ou l'authenticité des affects, mais bien de contrôler la conformité du projet marital avec l'idée d'égalité entre les sexes, valeur qui devient alors un critère implicite pour se voir offrir l'hospitalité en Belgique. On voit comment les agents défendent les frontières nationales en projetant la Belgique comme un espace civique de supériorité morale que les mœurs et les sexualités migrantes viendraient menacer.

Lorsque Laura Odasso a rencontré la population cible des administrations belges (des consulats, de l'Etat civil, de la police et de l'Office des étrangers), elle s'est trouvée dans des relations de proximité avec les interlocuteurs : les membres des couples binationaux se "libèrent » en effet face à la chercheure des expériences discriminantes et genrées vécues dans les bureaux de l'Etat. Les femmes nationales parlent d'un «paternalisme étatique ${ }^{29}$ écrasant qui dessine des formes stratifiées d'appartenance à la nation. Le fait d'être une femme ou un homme, d'être national ou étranger, d'avoir des origines et des appartenances religieuses, culturelles, communautaires, ou de classe - réelles ou perçues - joue un rôle décisif dans les interactions avec les représentants de l'Etat et a un impact non seulement sur les procédures, mais aussi sur le sens qu'ils attribuent à la citoyenneté. Ainsi, lorsque Laura Odasso cherche à comprendre comment les deux membres d'un couple binational perçoivent les contrôles que les agents de l'Etat leur réservent, elle est considérée comme un possible porte-parole qui aidera les couples dans leur situation en raison de sa qualification professionnelle, mais elle est aussi une femme. Le genre joue un rôle dans la relation particulière qui se construit pendant les entretiens et dans les détails narrés par ses interlocuteurs. Les femmes cherchent un appui chez la chercheure et sa compréhension féminine pour raconter, voire dénoncer, les traitements différentiels et abusifs que leur réservent certains agents de l'Etat et les effets que ces rencontres étatiques assez négatives ont eus sur leurs relations affectives et leur existence. C'est ainsi que les interactions enregistrées avec les femmes sont constamment ponctuées de phrases comme « Vu que vous êtes une femme, vous pouvez comprendre... » ou « Je ne sais pas si vous, en tant que femme, vous avez eu des expériences similaires ou l'impression qu'une administration vous traite comme une personne incapable d'agir seule et ayant besoin de protection » ou encore, "Qu'auriez-vous fait en tant que femme dans cette situation ? ». Lorsque Laura a rencontré les couples, elle a cherché à interroger les deux partenaires séparément, puis ensemble ; néanmoins, un déséquilibre dans les entretiens des hommes et des femmes existe, comme l'extrait de Samia le montre :

Quand ils m'ont demandé : « quand avez-vous couché avec votre mari pour la dernière fois ? Comment ? » Et tout... je me suis sentie mal. Je ne sais pas pour vous, mais cela me paraît absurde que le policier me demande cela. Je n'ai pas répondu. Et vous, qui êtes une femme comme moi, vous me comprenez, n'est-ce pas ? Vous auriez raconté à un inconnu votre vie privée et surtout votre vie sexuelle? (Entretien avec Samia, Bruxelles).

${ }^{29}$ M. JACKMAN, The Velvet Glove : Paternalism and Conflict in Gender, Class, and Race Relation, University of California Press, 1994. 
Quand Samia retourne la question à la chercheure, elle se positionne vis-à-vis d'elle sur un plan émotionnel d'égalité en raison du fait que la conversation se déroule entre deux femmes. Autant Samia n'est pas gênée de détailler sa relation intime à la chercheure, en allant souvent au-delà des questions axées principalement sur le rapport à l'administration, autant Samia ne conçoit pas la possibilité de dévoiler son intimité à des fonctionnaires masculins. Rien de similaire n'advient dans les récits des hommes : la complicité se joue sur un plan différent et, dans de rares cas, des hommes soulèvent le fait que la chercheure est étrangère comme eux dans le contexte belge afin de fournir des parallélismes pour mieux lui expliquer leurs propos.

L'autre volet exploré par les recherches de Laura Odasso concerne les entretiens avec des représentants du monde associatif qui soutient et aide les couples binationaux dans les démarches juridico-administratives et avec les policiers de trois équipes des polices (dites cellule « Recherche », " Mariages blancs » et « Services familles jeunesse et mariages forcés ») qui, à la ville de Bruxelles, interrogent les couples pour vérifier la véracité de leur union ou l'effectivité de leur cohabitation et recueillent les premières dénonciations des mariages de complaisance ou des violences intrafamiliales. Selon les entretiens conduits avec la police, la suspicion ${ }^{30}$ étatique affecte surtout les hommes étrangers dépourvus de titre de séjour ou ayant un titre précaire. Au contraire, les femmes, surtout belges, sont souvent traitées comme des actrices vulnérables et manipulables par ces hommes venus d'ailleurs avec le seul objectif de s'installer en Belgique. Toutefois, certaines femmes étrangères sont aussi décrites comme "menteuses "; l'âge des conjoints et leur ethnicité similaire ou différente participent à créer la suspicion et le modèle de fraude à l'institution du mariage qui sera à traquer. Dans ce cas, les policiers font appel à ce qu'ils pensent être évident pour la chercheure aussi. Ils partagent avec elle des représentations stéréotypées et naturalisées. Ils la poussent à adhérer à leur idée, car, en tant que femme et sociologue, elle pourra aisément comprendre « ce dont ils parlent ». Par exemple, lorsque la chercheure interroge les policiers de la cellule « Recherche » de la zone de police de Bruxelles-Ouest, ceux-ci, deux d'entre eux, proposent dans leurs explications narratives faites d'exemples et de catégories pratiques une vision répétitive et statique du genre et de ses effets, mais aussi du statut administratif et de l'origine nationale des membres des couples.

La situation classique, c'est un homme arabe sans-papiers, sans titre de séjour qui est sur le territoire belge depuis des années ou est rentré irrégulièrement. C'est clair que là, nous ne nous laissons pas trop faire par l'histoire d'amour. Ses démarches préalables sont importantes, ces hommes ont souvent fait d'autres demandes... On est obligés d'y voir plus clair, de demander. Elle, par contre, est une belle jeune Belge. Ils sont tombés amoureux et rapidement se marient. Ça, on le voit tout le temps. Puis t'as le Belge d'origine qui ramène sa femme et là, t'as un tout autre scénario. Aux femmes belges, on demande si elles ont reçu de l'argent, si elles sont sûres de leur choix, aux hommes belges pas toujours... Et puis on pose toutes les questions de connaissance et de routine, on a le feeling désormais. Des nationalités reviennent dans les mêmes scénarios, mais je dirai que c'est les hommes qui n'ont pas d'autres possibilités qui

${ }^{30}$ Cette suspicion n'est pas non plus absente dans le monde associatif, toutefois cet aspect mériterait un approfondissement particulier qui ne peut pas trouver place ici. 
cherchent à se régulariser via le mariage. On le sait et on le limite (Entretien avec Ernest, cellule « Recherche », Zone de Police Bruxelles-Ouest).

Si les policiers affirment que d'autres cas existent, ils tendent, néanmoins, à narrer toujours des dynamiques visant à faire émerger une certaine masculinité et une certaine féminité. Dans les interactions, le genre de la chercheure sera un outil qui facilite la transmission de l'information, comme ces observations récurrentes le suggèrent : « vous aussi vous voudriez un mari qui connaît vos goûts, n'est-ce pas ?», « Toutes les femmes veulent une fête de mariage ! Vous?» ou «Pensez-vous pouvoir aimer quelqu'un qui a plus de dix ans que vous? Comment planifier une vie et des enfants?».

Dans les deux situations prises pour exemple - les entretiens avec les membres de couple et ceux avec la police -, la chercheure est confrontée d'une manière différente à des propos qui lui semblent trop tranchants et doit, en opérant un effort ontologique, jouer entre le «soi-individu », qui possède une idée sur la question, et le « soi-chercheure ", qui veut comprendre les logiques qui poussent ces policiers à appréhender le genre des usagers de cette manière. Ce travail de participation et de mise à distance opérée sur la « chercheure-individu » est un des questionnements opérés pendant les parcours d'investigation. Dans ces cas rapportés et dans de nombreuses autres situations discutées pendant le projet belgo-argentin, les chercheures ont dû faire face à des « dilemmes moraux » que nous aborderons dans la section suivante.

\section{Observer les contrôleurs : des dilemmes moraux}

Si l'association entre la figure de l'anthropologue et celle de l'espion existe de longue date, cette affinité s'intensifie en terrain étatique. A de nombreuses occasions, le travail de terrain de Sabrina Calandrón avec la police de Buenos Aires fait écho à cette association faite par les « ethnographiés ». Le fait de noter des éléments sur le cahier de terrain attirait l'attention des agents qui demandaient ironiquement et avec suspicion si la chercheure faisait partie des Asuntos Internos (Affaires intérieures). En Argentine, les Asuntos Internos désignent le tribunal de justice interne pour les policiers où sont traitées les dénonciations vis-à-vis des agents en tant que fonctionnaires publics. La question montre que les agents avaient le sentiment d'être inspectés ou menacés par la chercheure, non par sa présence, mais bien parce qu'elle prenait des notes. Ce sentiment d'être observé a dû également être apprivoisé par les agents communaux durant la présence prolongée de Maïté Maskens dans leurs bureaux. Cette dernière arrivait à la même heure qu'eux et repartait à la fin de la journée de travail. Une des manières de désamorcer le malaise provoqué par sa présence et son regard attentif fut le recours à l'humour. C'est ainsi que Gaston, un employé de la commune approchant de la soixantaine, repris par Yvonne, sa collègue, alors qu'il parlait de manière vulgaire lui rétorqua : «Quoi ? Tu crois que parce que Maïté est là, je vais changer quoi que ce soit dans ma manière d'être, là tu te trompes ! ». Il accompagna ses paroles d'un geste provocateur en dirigeant vers Yvonne un doigt d'honneur.

Dans cette section, nous interrogeons les spécificités méthodologiques inhérentes à l'étude d'acteurs particuliers, ceux qui possèdent un certain pouvoir : celui d'interroger, d'interpréter, de contrôler et de prendre des décisions qui concernent la vie - et même la vie intime - d'autres individus. La décision des agents aura des conséquences parfois 
décisives sur la vie des personnes dont ils traitent les « dossiers ». Ce genre de terrain rompt avec certaines formes de misérabilisme qui ont marqué la recherche en sciences sociales depuis ses débuts : l'étude des "sauvages », des victimes et des pauvres, menée par une classe d'intellectuels dominants. Cette focalisation du regard vers les puissants n'est pas sans conséquence sur les manières de mener une recherche. Que faire lorsque les attitudes et les mises en œuvre des politiques publiques déplaisent au chercheur/à la chercheure ? Que devient l'idéal d'empathie, injonction, pour autant, au cœur de la méthode ethnographique ? Comment travailler avec des gens dont les pratiques nous rebutent? Ces tensions et ces ambiguïtés provoquent bien souvent des dilemmes moraux que le chercheur doit résoudre.

Nous montrerons sur nos trois terrains singuliers comment se déclinent ces dilemmes moraux propres à la conduite d'une recherche sur l'Etat en train de se faire. Ici, un exemple du travail de Maïté Maskens vient donner corps à ces difficultés de positionnements. Autorisée à être présente lors des entretiens réalisés par les agents lorsque les couples étaient suspectés de mariage de complaisance depuis plusieurs mois, la chercheure était de plus en plus encouragée à y participer. Les agents se tournaient souvent vers elle à la fin d'un entretien pour lui demander si elle avait encore des questions. D'abord très surprise par ce glissement de rôle, la chercheure répondait à chaque fois par la négative afin de pas alimenter la confusion. Lors d'un entretien avec un couple camerounais, Maïté profita de l'espace qui lui était ménagé petit à petit pour intervenir dans ce qui lui paraissait être une injustice relevant de l'ignorance bureaucratique ${ }^{31}$.

L'histoire du couple dont les agents tentaient tant bien que mal de retracer les lignes était compliquée, elle impliquait de nombreux allers-retours entre l'Europe et l'Afrique, différents mariages et enfants, des trahisons et surtout des conceptions culturelles très différentes de l'autorité. Pour le dire vite, l'homme du couple s'était résigné à accepter l'avis de son père défavorable à l'union avec la femme qu'il aimait, car celle-ci était déshonorée par un enfantement précoce. Pour Gaston, l'agent de l'Etat alors présent, cette soumission était incompréhensible et il dira à plusieurs reprises : «M'enfin, vous faites toujours ce que votre père dit ! » avec un ton surpris, voire désapprobateur. Ce n'est qu'à la mort du père que l'homme assumera son choix. Pour Gaston, ce récit allait contre ses convictions profondes concernant la liberté et la modernité. Il attendait des récits que les migrants y apparaissent comme des individus libérés du poids de la famille et des généalogies; autonomes dans leur choix. Cela faisait partie de ses critères implicites d'évaluation. A cette soumission incomprise venait s'ajouter la complexité de l'histoire qui éveillait davantage les soupçons de Gaston qui avait le sentiment que toute cette complexité cachait quelque chose. Le décor de la rencontre du couple dans le chef de l'homme était la ville de Douala. Lorsque ce fut au tour de la femme de livrer son récit de la rencontre, celle-ci se référait à Bépanda comme lieu qui a vu naître leur histoire d'amour. Lorsque Gaston entendit cette localité, il ne pouvait plus se tenir sur sa chaise, il se tortillait en me faisant de grands yeux. Pour lui, le diable est dans le détail et il tenait dans ces deux appellations différentes la preuve de l'inauthenticité (Douala vs Bépanda) des récits. De mon côté, convaincue de la véracité de l'histoire que je venais d'entendre - je ne pensais pas que l'on puisse inventer une telle complexité - et puis plus informée sur les

${ }^{31}$ D. Graeber, The Utopia of the Rules, New York, Melville House, 2015. 
manières de dire les affects sur le continent africain, je m'autorisai à intervenir lorsque la femme fut sortie du bureau. Je demandai à Ana, qui avait retranscrit l'entretien, de regarder sur Internet si Bépanda n'était pas une autre manière de nommer Douala et après quelques clics sur internet, elle nous informa que Bépanda était un quartier de Douala. Cela conduit à éteindre en partie les soupçons de Gaston, un peu déçu maintenant (Carnet de terrain, Bruxelles, 2013).

Dans cet exemple, on voit que la chercheure n'a pu se retenir d'intervenir lors de la phase d'observation participante, faisant fi du principe de neutralité axiologique afin de gérer la difficulté d'être témoin de l'ignorance et donc potentiellement de la violence bureaucratique.

Le travail ethnographique, l'observation participante et la situation d'entretien sont traversés par des relations de pouvoir qui influent sur le comportement des acteurs et qui demandent à la chercheure une prise de décision éthique. Deux questions nous ont travaillées au corps et au cœur : 1) que faire face au désaccord et à la colère que ces injustices provoquaient en nous ? 2) comment faire pour ne pas être ni se sentir complices de ces injustices ? La réponse n'est pas simple. En premier lieu, nous sommes revenues sur la question de l'empathie, au cœur de la pratique ethnographique : bien que la capacité de se mettre à la place de l'autre soit un exercice pertinent dans la production des données, il ne peut en aucun cas constituer une obligation. Des sentiments négatifs surgissent parfois - l'anthropologue ou le sociologue n'est pas plus vertueux qu'un humain ordinaire - et il ne faut pas les refouler au nom d'une injonction disciplinaire à l'empathie, d'autant que ces affects nous permettent aussi de problématiser davantage le terrain. De plus, dans ces rencontres étatiques qui confrontent des individus aux intérêts divergents, parfois conflictuels, il est pratiquement assez difficile de se mettre à la place de chacun des acteurs. En deuxième lieu, accepter que notre présence a de toute façon une influence sur la vie sociale que nous observons est un préalable incontournable à la recherche en sciences sociales depuis les écrits de Georges Devereux ${ }^{32}$. Il faut donc considérer cette « interférence » comme une ressource plus que comme un échec de la recherche en sciences sociales. En ce sens, la possibilité d'exprimer des propos différents de ceux de nos informateurs constitue une manière de co-construire le savoir et de prendre nos interlocuteurs au sérieux ${ }^{33}$; et pourquoi pas, en les poussant dans leur retranchement à certaines occasions. En troisième lieu, il a fallu maintenir la certitude que si notre proximité avec les agents pouvait produire de la confusion au sein de la population cible des agents - qui pouvaient donc penser que nous appuyons leurs actes - cela ne changeait en revanche pas radicalement la pratique de la police et des bureaucrates. En quatrième lieu, le respect du pacte ethnographique passé avec les interlocuteurs doit primer en toutes circonstances sur la dénonciation d'injustices ; la

${ }^{32}$ G. Devereux, De l'angoisse à la méthode dans les sciences du comportement, Paris, Payot, 1980.

${ }_{33}$ Voir B. Latour, Changer de société, refaire de la sociologie, Paris, La Découverte, 2006, p. 19 et 22 et Martina Avanza dans son travail au sein du parti xénophobe de la Ligue $d u$ Nord en Italie (M. AvanZA, « Comment faire de l'ethnographie quand on n'aime pas « ses indigènes »? Une enquête au sein d'un mouvement xénophobe ", in D. FASSIN et A. BENSA, Les politiques de l'enquête, Paris, La Découverte, 2008, p. 41-58). 
confidentialité promise ne peut être rompue au nom d'idéaux d'égalité et de justice car cela reviendrait à exposer des acteurs qui nous ont donné leur confiance, sapant ainsi la base même du travail de terrain. Enfin, en dernier lieu, faire une enquête en sciences sociales ne nous empêche pas de nous engager dans des organisations sociales ou politiques hors travail de terrain où nous pouvons travailler à rendre les actions étatiques plus justes et équitables en dénonçant les mécanismes - volontaires et involontaires - à la base des injustices dont nous avons été parfois les témoins.

\section{Conclusions et perspectives}

L'article a mis en lumière la pertinence et la fécondité d'une étude croisant la problématique du genre avec les logiques d'Etat, et la richesse d'une analyse comparative transatlantique. Cette approche nous a permis d'esquisser les contours d'une théorie réflexive et pragmatique de l'Etat au prisme du genre. L'action de la police, des administrations en charge du contrôle de l'immigration familiale, ainsi que l'expérience qu'en font les usagers sont traversées par des rapports de genre qui tendent à reproduire des représentations figées de la féminité et de la masculinité. Ces représentations traversent l'Atlantique et, de l'Argentine à la Belgique, apparaissent dans des contextes politiques et institutionnels différents qui produisent des formes de citoyennetés inégales. Tant dans le travail d'enquête que dans les résultats d'analyse, des similitudes émergent.

Primo, il a été possible de mettre en évidence les logiques genrées de l'Etat qui se manifestent dans le contrôle sous deux formes. D'une part, des conceptions du genre gouvernent les relations entre les agents de l'Etat et organisent leurs relations, leurs hiérarchies et la distribution du pouvoir. D'autre part, l'idéologie de genre s'exprime dans les actions vis-à-vis de la population, des usagers de ces administrations. Dans ces interactions entre agents et usagers, les normes juridiques et légales s'appliquent en étant travaillées par des conceptions hégémoniques du genre. La norme abstraite est interprétée lors de l'application pratique au moyen d'idées figées sur la place des femmes et des hommes dans la famille, dans le couple et dans la société à laquelle elles/ils appartiennent ou désirent appartenir.

Secundo, les échanges des trois chercheures au sujet de leurs analyses mettent en lumière la question de la perméabilité de l'Etat comme champ d'étude empirique. Etre une femme a permis un accès au terrain marqué par des présupposés de sensibilité, de docilité et d'empathie genrée. Les agents étatiques ont bien souvent vu dans les chercheures en sciences sociales des sortes d'assistantes sociales, soulignant ainsi les aptitudes prétendument féminines à la sensibilité, à l'écoute et à la bienveillance ${ }^{34}$. En

${ }^{34}$ L'idée d'une délicatesse proprement féminine est répandue dans ces arènes étatiques. Dans l'étude de la police menée par Sabrina Calandrón, cette idée s'est matérialisée à différentes occasions dans des interactions : d'abord lorsque les agents s'excusaient devant la chercheure d'employer un langage « grossier» qui n'était pas approprié « devant une demoiselle ». Cette notion prend corps également dans les échanges lorsque les policiers considèrent la violence des femmes policières, perçue comme moins intense que celle des hommes. Ceci, en dépit de l'exemple d'une agente féminine correctement entraînée qui a pu arrêter, immobiliser et transférer un détenu alors que ses deux compagnons, plus âgés, obèses et maladroits, réussirent à peine à maintenir immobilisé au sol un autre détenu, après que ce dernier ait cassé une fenêtre 
raison de cette identification, dans de nombreuses situations, les chercheures étaient invitées à écouter et à donner leur opinion sur des situations visant la population considérée.

L'appartenance au monde académique et l'empathie ont été deux clés d'accès au terrain étatique, configurant les échanges ethnographiques de manière spécifique. Dans plusieurs endroits, l'effort d'observation s'est traduit en un travail de contrôle de celui qui contrôle dans un espace social où exercer le rôle de contrôle hiérarchise les acteurs. Ce n'est donc pas une surprise si ce travail de terrain et son corollaire méthodologique - l'injonction disciplinaire à l'empathie - furent marqués par bien des dilemmes moraux. Sur ces trois terrains, les chercheures ont affronté le même dilemme moral et épistémologique, celui de rendre intelligible le point de vue de groupes dont elles ne partageaient pas l'idéologie ou qui nous considèrent, nous, les enquêtrices, comme une menace politique ou juridique. Bien qu'il ne s'agisse pas ici d'un problème nouveau pour les sciences sociales ${ }^{35}$, nous espérons avoir pu contribuer à son renouvellement en terrain étatique. L'analyse croisée des pratiques étatiques en Argentine et en Belgique, les descriptions de relations de pouvoir (entre les chercheurs et les agents de l'Etat et entre ces derniers et leurs usagers), les questionnements sur la position de la chercheure et les analyses de ses perceptions, de ses émotions et de ses dilemmes contribuent à montrer que l'Etat, par le truchement de ses agents, agit bien au travers d'idées, d'attentes et d'idéologies du genre que les sciences sociales se doivent de déconstruire sans cesse. Dans ces espaces, le risque de l' " évidence » du masculin et du féminin a des conséquences très pratiques sur la vie des individus, mettant ainsi la notion de citoyenneté au conditionnel.

et donné un coup à un autre policier durant la lutte. Même s'il est évident que la condition physique et la capacité d'utiliser des armes ne dépendent pas de l'identité de genre, le discours policier insiste sur le caractère plus faible des femmes policières (S. CALANDRÓN, " Gender expressions, morality and the use of physical force by the Argentine police », European Journal of Policing Studies, 4/4, 2017, p. 405-422).

${ }^{35}$ F. GinsBurG, «Quand les indigènes sont nos voisins », L'Homme, 1992, 32/121, p. 129142 ; M. Huggins et M. Glebbeek, «Women Studying Violent Male Institutions : CrossGendered Dynamics in Police Research on Secrecy and Danger », Theoretical Criminology, 7/3, 2003, p. 363-387. 